\title{
Crescimento e produtividade do pinhão-manso em função do espaçamento e irrigação
}

\author{
Ana C. 0. Horschutz ${ }^{1}$, Marconi B. Teixeira ${ }^{1}$, José M. Alves ${ }^{1}$, \\ Fabiano G. Silva ${ }^{1} \&$ Nelmício F. da Silva ${ }^{1}$
}

\begin{abstract}
RESU M 0
O bjetivou-se, com este trabalho, avaliar o efeito do espaçamento e da complementação hídrica no crescimento e produtividade do pinhão-manso. 0 experimento foi conduzido na área experimental do Instituto Federal Goiano, campus Rio Verde,GO, no período de 12/09/2009 a 30/04/2011, no delineamento de blocos casualizados com três repetições, os fatores foram sete espaçamentos e duas condições hídricas, cada parcela constituída de 16 plantas, sendo as quatro plantas centrais a área útil. Aos 60 dias após o transplantio das mudas foram feitas, bimensalmente, as avaliações dos parâmetros al tura de planta, número de ramificacões, área foliar e produtividade. 0 fator espaçamento não apresentou diferença significativa para os parâmetros altura, ramificação e produtividade e a complementação hídrica teve efeito significativo para altura aos 240 dias após transplantio. A produção de sementes em sequeiro foi maior no espaçamento $3 \times 3$ e com complementação hídrica no espaçamento $4 \times 4$.
\end{abstract}

Palavras-chave: Jatropha curcas L., biodiesel, oleaginosa, emissor

\section{Growth and productivity of physic nut as a function of plant spacing and irrigation}

\begin{abstract}
The objective of this study was to evaluate the effect of plant spacing and water supplementation on growth and productivity of physic nut (Jatropha). This research was carried out at the experimental area of the Instituto Federal G oiano - Rio Verde during the period 12/09/2009 to 30/04/2011, in a randomized block design with three replications. The factors consisted of seven plant spacings and two water conditions, each plot consisted of 16 plants, and four central plants were considered for data collection. At 60 days after transplanting of seedlings, fortnightly the evaluation of the parameters plant height, number of branches, leaf area and productivity was carried out. The plant spacing showed no significant difference for height, branching and productivity, while the water supplementation had significant effect on height at 240 days after transplanting. Seed production under rainfed conditions was higher in $3 \times 3 \mathrm{~m}$ spacing and with complementary water in $4 \times 4 \mathrm{~m}$ spacing.
\end{abstract}

Key words: Jatropha curcas L., biodiesel, rapeseed, emitter 


\section{INTRODUÇÃO}

O pinhão-manso é uma espécie perene pertencente à família das Euforbiáceas, a mesma família da mamona, mandioca e seringueira, amplamente distribuído em áreas tropicais e subtropicais com potencial para a produção de biocombustível (Laviola \& Dias, 2008).

A espécie Jatropha curcas L. caracteriza-se pela fácil propagação, rápido crescimento, elevado teor de óleo, podendo ser cultivada em áreas de baixa e alta precipitação. Sua fase reprodutiva se inicia a partir do décimo mês após o plantio, período em que ocorre aumento progressivo de produção até o terceiro ou quarto ano, quando se estabiliza, podendo chegar a quatro metros de altura e manter o período produtivo por até 40 anos (Arruda et al., 2004; Oliveira et al., 2007; Sujatha et al., 2009; Martins et al., 2010).

O interesse comercial no Brasil, pela cultura, foi despertado por apresentar características desejáveis tanto na renovação da base energética nacional como na agricultura familiar, favorecendo a permanência do homem no campo, por se tratar de uma cultura com amplo potencial agrícola, destacando-se pela alta produtividade e qualidade satisfatória do óleo para produção de biodiesel (Francis et al., 2005; Teixeira, 2005).

O desenvolvimento do pinhão-manso é favorecido em regiões tropicais cuja precipitação anual esteja acima de 600 mm e, acima dos $1000 \mathrm{~mm}$ anuais, o nível ideal de precipitação para que possam ser atingidas altas produtividades. Avaliando a primeira colheita, Drumond et al. (2010) obtiveram produtividade de $871 \mathrm{~kg} \mathrm{ha}^{-1}$ em plantas irrigadas e, em regime normal de chuvas (sequeiro), $246 \mathrm{~kg} \mathrm{ha}^{-1}$, a produção é drasticamente afetada com valores anuais inferiores a $600 \mathrm{~mm}$.

Para se obter alta produtividade de frutos, a planta necessita de água e exige solos férteis e com boas condições físicas. Logo, a irrigação, a correção da acidez e da fertilidade do solo, são fatores decisivos para se obter sucesso e lucratividade com esta cultura (Chaves et al., 2009; Schiavo et al., 2010).

São poucas as informações sobre os aspectos agronômicos básicos da cultura como os mecanismos de tolerância à seca e, por se tratar de uma espécie silvestre não melhorada geneticamente, ocorrem dificuldades para seu cultivo (Nery et al., 2009). Em referência à irrigação, deve-se optar pela microaspersão no lugar da irrigação por gotejamento em solos muito permeáveis, pelo risco da não formação adequada do bulbo úmido mínimo necessário para as culturas (Openshaw, 2000). Desta forma, torna-se imprescindível conhecer as características do modelo de microaspersor selecionado para um dimensionamento correto e manejo adequado da irrigação na área de cultivo.

Tendo em vista o exposto objetivou-se, com este trabalho, avaliar o efeito do espaçamento e da complementação hídrica no crescimento e na produtividade do pinhão-manso, na região do sudoeste goiano.

\section{MATERIAL E MÉTODOS}

O experimento foi instalado no município de Rio Verde, GO, na área experimental do Instituto Federal Goiano (IFGoianocampus Rio Verde), e conduzido no ano agrícola de 2010/2011. A região se caracteriza por apresentar precipitação média anual em torno de $1740 \mathrm{~mm}$, clima tropical quente com estações chuvosa e seca definidas, temperatura média anual em torno de 20 a $25^{\circ} \mathrm{C}$ e relevo relativamente plano, numa altitude de 748 m (Bernardi et al., 2003).

Anterior à instalação do experimento, foi feita uma amostragem do solo nas camadas de $0-20$ e $20-40 \mathrm{~cm}$ de profundidade para análise físico-química (Tabela 1). Amostras indeformadas foram retiradas nas mesmas profundidades para confecção da curva de retenção de água no solo em porcentagem de volume, no intervalo de tensão entre 5 e 1.500 $\mathrm{kPa}$, ajustadas à equação de Genuchten (1980), utilizando-se o programa RETC (Genuchten, 1991).

De acordo com a análise química do solo, a saturação de base (v) do solo foi elevada de 52,38\% para 70\%, utilizando-se o calcário dolomítico (PRNT 70\%), com base no cálculo de necessidade de calagem:

$$
\mathrm{Nc}=\frac{(\mathrm{V} 2-\mathrm{V} 1) \times \mathrm{T}}{10 \times \mathrm{PRNT}}
$$

As mudas de pinhão-manso foram produzidas no dia 28/09/ 2009 com sementes provenientes da EPAMIG, em sacos de polietileno com capacidade de $1000 \mathrm{~mL}$ com mistura de terra e uma concentração de $20 \%$ de matéria orgânica (Horschutz et al., 2008), sendo semeadas em cada recipiente duas sementes; 15 dias após a emergência das plântulas fez-se o desbaste, permanecendo as plantas mais desenvolvidas e centralizadas. As mudas foram transplantadas para o campo três meses após a semeadura.

O preparo do solo constituiu de aração, gradagem e nivelamento da área experimental. As mudas foram transplantadas em covas nas dimensões de 30 x 30 x $30 \mathrm{~cm}$. A adubação nitrogenada foi de $120 \mathrm{~kg} \mathrm{ha}^{-1}$ de ureia aplicados em cobertura aos 30 e 90 dias após o transplantio das mudas.

$\mathrm{O}$ delineamento experimental utilizado foi em bloco casualizados, segundo um esquema fatorial 7 x 2, com três repetições. Os fatores foram constituídos de sete espaçamentos $(2 \times 3,2 \times 4,3 \times 3,3 \times 4,4$ x 4, 5 × 3 e 5 x 4) e duas condições hídricas (com e sem irrigação). Cada parcela foi constituída de 16 plantas, sendo as quatro plantas centrais a área útil.

Utilizou-se o sistema de irrigação localizada com microaspersores de fluxo turbulento modelo Hadar 7110 (Naan Dan Jain Brasil) com vazão de 29,0 L h-1 a 1,0 bar, diâmetro molhado igual a $6,0 \mathrm{~m}$, com microaspersor apoiado em haste a $0,25 \mathrm{~m}$ do solo e $0,40 \mathrm{~m}$ do caule da planta, utilizando-se uma lateral de microaspersores por linha de plantas (um microaspersor por planta).

Tabela 1. Atributos químicos e físicos do solo da área de implantação do experimento

\begin{tabular}{|c|c|c|c|c|c|c|c|c|c|c|c|c|c|}
\hline \multirow{2}{*}{$\begin{array}{c}P \\
\mathrm{mg} \mathrm{dm}^{-3}\end{array}$} & \multirow{2}{*}{$\begin{array}{l}\text { M.O. } \\
\mathrm{g} \mathrm{dm}^{-3}\end{array}$} & \multirow{2}{*}{$\begin{array}{c}\mathrm{pH} \\
\mathrm{H}_{2} \mathrm{O}\end{array}$} & $\mathrm{K}^{+}$ & $\mathrm{Ca}^{++}$ & $\mathrm{Mg}^{++}$ & $\mathrm{H}^{+} \mathrm{Al}$ & 5 & $T$ & V \% & \multirow[t]{2}{*}{$\mathrm{AL}^{++}$} & \multicolumn{3}{|c|}{ Textura $\left(\mathrm{g} \mathrm{kg}^{-1}\right)$} \\
\hline & & & \multicolumn{7}{|c|}{$\mathrm{mmol} \mathrm{dm}^{-3}$} & & Argila & Limo & Areia \\
\hline 33,229 & 54,61 & 6,2 & 7,1 & 36,8 & 23,2 & 61,0 & 67,1 & 128,2 & 52,3 & 0,0 & 382,3 & 148,9 & 468,8 \\
\hline
\end{tabular}

M.O. - matéria orgânica, $S$ - soma de bases, $T$ - capacidade de troca catiônica, $V$ - saturação de base 
Ensaios de uniformidade de distribuição de água foram realizados utilizando-se o coeficiente de uniformidade de Christiansen (CUC, \%) para verificar o funcionamento do sistema, ao longo do ensaio.

O manejo da irrigação foi realizado via dados climáticos com uso da equação de Penman-Monteith para cálculo da evapotranspiração de referência $\left(\mathrm{ETo}, \mathrm{mm} \mathrm{d}^{-1}\right)$. Para o cálculo da evapotranspiração da cultura (ETc, $\left.\mathrm{mm} \mathrm{d}^{-1}\right)$, utilizou-se o valor do coeficiente de cultivo igual a $1,0(\mathrm{Kc}=1,0)$. A quantidade de água aplicada para os tratamentos com suplementação hídrica foi o suficiente para suprir a demanda hídrica da cultura, com turno de rega a cada quatro dias.

A partir dos 60, 120, 180, 240, 300, 360, 420 e 480 dias após o transplantio das mudas, foram feitas as seguintes avaliações:

A altura da planta foi determinada na região compreendida entre o nível do solo e o meristema apical da haste principal, com o auxílio de trena.

O número de ramificações por planta foi determinado pela contagem direta.

A area foliar foi definida em uma folha completamente expandida pela equação $A=L^{1,87}$, indicada por Severino (2007), em que A corresponde à área foliar em $\mathrm{cm}^{2} \mathrm{e} \mathrm{L}$, à largura da folha, que foi obtida com uma régua graduada em centímetros.

Para a produção os frutos com coloração amarela, com manchas marrom e marrom a preto, foram coletados das quatro plantas centrais das parcelas a partir dos 210 dias após o transplantio (DAT) das mudas e posteriormente de $15 \mathrm{em} 15$ dias, até os 480 DAT. Os frutos foram armazenados em sacos de papel e levados à estufa a $55^{\circ} \mathrm{C}$ até atingir peso constante; após este período a casca e a semente foram separadas e pesadas em balança de precisão.

Os dados foram submetidos à análise de variância e as médias comparadas pelo teste de Tukey a nível de 0,05 de probabilidade, com o auxílio do software SISVAR.

\section{RESULTADOS E DISCUSSÃO}

Apesar de o parâmetro espaçamento não apresentar significância estatística, observa-se que o espaçamento mais adensado ( $2 \times 3)$, com $6 \mathrm{~m}^{2}$ por planta, apresentou as menores médias, exceto aos 120 DAT (Tabela 2), podendo este resultado ter ocorrido em razão da menor disponibilidade de nutrientes para as plantas por $\mathrm{m}^{2}$.

Os resultados em relação ao espaçamento diferem dos obtidos por outros autores, que afirmam que os menores espaçamentos apresentam as maiores alturas. Pinto Júnior \& Almeida (2009) concluíram, trabalhando com o desenvolvimento inicial do pinhão-manso em diferentes espaçamentos, que as maiores médias para a altura do pinhão-manso ocorreram nos espaçamentos de 3 x 0,50 e 3 x 0,75 $\mathrm{m}$ aos 80, 150 e 220 DAT com altura de 30,$20 ; 35,56$ e $36,15 \mathrm{~cm}$, respectivamente. O pinhãomanso crescido sob condições climáticas da Tailândia, atingiu altura entre 83 a $110 \mathrm{~cm}$ aos 14 meses de idade (Ratree, 2004).

Nunes et al. (2008) afirmam, trabalhando com o desenvolvimento vegetativo do pinhão-manso no oeste da Bahia, aos 360 DAT, que a altura média encontrada foi de 120 $\mathrm{cm}$, no espaçamento de $3 \times 2 \mathrm{~m}, 30,72 \%$ menor em altura que as plantas estudadas neste experimento com a mesma idade e espaçamento.

A partir dos 240 DAT verificou-se efeito significativo para a condição hídrica com as plantas com complementação hídrica apresentando maior altura (Tabela 3) resultados que indicam que as plantas irrigadas têm um desenvolvimento vegetativo maior, sendo necessário um espaçamento também maior para que não haja competição nutricional e espacial.

Evangelista et al. (2011) constataram, trabalhando com irrigação em pinhão-manso, que plantas em sequeiro aos 412 DAT tinham altura média de 2,37 $\mathrm{m}$ e as plantas irrigadas com uma lâmina de $80 \%$ da capacidade de campo apresentaram altura média de 2,70 m. Para o presente trabalho foi observado, aos

Tabela 2. Altura de plantas (cm) do pinhão-manso cultivado em diferentes espaçamentos

\begin{tabular}{|c|c|c|c|c|c|c|c|c|}
\hline \multirow{3}{*}{ Espaçamento } & \multicolumn{8}{|c|}{ Atura de plantas $(\mathrm{cm})$} \\
\hline & 60 DAT* & 120 DAT & 180 DAT & 240 DAT & 300 DAT & 360 DAT & 420 DAT & 480 DAT \\
\hline & \multicolumn{8}{|c|}{ Médias } \\
\hline $2 \times 3$ & $47,79 \mathrm{a}$ & $80,25 a$ & $121,77 \mathrm{a}$ & $151,06 \mathrm{a}$ & $165,82 \mathrm{a}$ & $173,20 \mathrm{a}$ & $179,60 \mathrm{a}$ & $189,00 \mathrm{a}$ \\
\hline $2 \times 4$ & $58,87 a$ & $84,87 \mathrm{a}$ & $135,45 a$ & $169,12 \mathrm{a}$ & $186,18 \mathrm{a}$ & $194,70 \mathrm{a}$ & $215,41 a$ & $217,83 a$ \\
\hline $3 \times 3$ & $57,38 \mathrm{a}$ & 85,62 a & $138,91 \mathrm{a}$ & $162,40 \mathrm{a}$ & $177,63 \mathrm{a}$ & $189,25 a$ & $203,13 a$ & $205,05 \mathrm{a}$ \\
\hline $3 \times 4$ & $46,78 \mathrm{a}$ & 76,75 a & $133,13 a$ & $156,21 \mathrm{a}$ & $176,89 a$ & $187,23 a$ & $201,76 \mathrm{a}$ & 207,07 a \\
\hline $4 \times 4$ & $55,08 \mathrm{a}$ & $83,20 \mathrm{a}$ & $138,62 a$ & $178,45 a$ & $192,59 a$ & $199,66 \mathrm{a}$ & $212,54 \mathrm{a}$ & $217,33 \mathrm{a}$ \\
\hline $5 \times 3$ & $58,45 a$ & 83,83 a & $126,91 \mathrm{a}$ & $166,66 \mathrm{a}$ & $179,00 \mathrm{a}$ & $185,16 \mathrm{a}$ & 198,12 a & 203,62 a \\
\hline $5 \times 4$ & $56,54 a$ & 79,33 a & $122,87 \mathrm{a}$ & $154,08 \mathrm{a}$ & $173,72 a$ & $183,54 \mathrm{a}$ & 199,16 a & $204,16 \mathrm{a}$ \\
\hline CV (\%) & 21,70 & 14,88 & 12,54 & 14,62 & 12,83 & 12,71 & 12,2 & 11,36 \\
\hline
\end{tabular}

* DAT - Dias após o transplantio; Médias seguidas de mesma letra minúsculas nas colunas não diferem entrei si pelo teste de Tukey, a nível de 0,05 de probabilidade

Tabela 3. Altura de plantas $(\mathrm{cm})$ do pinhão-manso cultivado em duas condições hídricas

\begin{tabular}{lcccccccc}
\hline \multirow{2}{*}{ Condição hídrica } & \multicolumn{7}{c}{ Atura de plantas (cm) } \\
\cline { 2 - 9 } & 60 DAT* & 120 DAT & 180 DAT & 240 DAT & 300 DAT & 360 DAT & 420 DAT & 480 DAT \\
\cline { 2 - 9 } & & \multicolumn{7}{c}{ Médias } \\
Sequeiro & $55,60 \mathrm{a}$ & $82,06 \mathrm{a}$ & $127,10 \mathrm{a}$ & $152,33 \mathrm{~b}$ & $169,48 \mathrm{~b}$ & $178,05 \mathrm{~b}$ & $192,76 \mathrm{~b}$ & $197,09 \mathrm{~b}$ \\
Comple. Hídrica & $53,22 \mathrm{a}$ & $81,66 \mathrm{a}$ & $135,09 \mathrm{a}$ & $172,80 \mathrm{a}$ & $188,19 \mathrm{a}$ & $195,88 \mathrm{a}$ & $209,45 \mathrm{a}$ & $215,50 \mathrm{a}$ \\
CV (\%) & 21,70 & 14,88 & 12,54 & 14,62 & 12,83 & 12,71 & 12,2 & 11,36 \\
\hline
\end{tabular}

* DAT - Dias após o transplantio; Médias seguidas de mesma letra minúsculas nas colunas, não diferem entrei si pelo teste de Tukey, a nível de 0,05 de probabilidade 
420 DAT, que a média de altura em sequeiro foi de $192,76 \mathrm{~cm}$ e de 209,45 cm, com complementação hídrica.

Apesar de os fatores não apresentarem diferenças significativas nota-se que o menor espaçamento $(2 \times 3)$ apresentou as menores médias (Tabela 4), podendo este fato ter ocorrido pela competição de espaço entre as plantas e menor disponibilidade de nutrientes.

Evangelista et al. (2011) encontraram, trabalhando com resposta de crescimento do pinhão- manso à aplicação de diferentes lâminas de irrigação, o valor médio de 5,30 ramos aos 412 DAT no espaçamento de 4 x 2 em sequeiro.

Pode-se observar, após 270 DAT, que as plantas com complementação hídrica obtiveram maior altura, Tabela 5, e menor número de ramos que as plantas submetidas a sequeiro.

Plantas de pinhão-manso na fase inicial de desenvolvimento apresentaram, em média, 3 hastes por planta aos 220 DAT, nos espaçamentos de 3 x 0,5 e 3 x 0,75 (Pinto Júnior \& Almeida, 2009). Santos (2010) concluiu, estudando os caracteres agronômicos da cultura do pinhão-manso em diferentes espaçamentos, adubações e poda no estado do Rio de Janeiro, que nos espaçamentos de $2 \times 1,5,2 \times 2,3 \times 2$ e 2 × 3,5 m aos 15 meses o número médio de hastes foi de 5,45, 4,95, 4,70 e 5,80, respectivamente, e aos 22 meses o número médio de hastes foi de 7,30, 9,25, 9,95 e 8,20, respectivamente.

Para os fatores espaçamento e complementação hídrica, houve diferença significativa aos 420 DAT (Tabela 6), e aos 240 DAT (Tabela 7), respectivamente.

Aos 420 DAT as maiores médias para área foliar foram observadas nos espaçamentos 3 x 4 e 5 x 4, com valores de 151,11 e 154,63 $\mathrm{cm}^{2}$, respectivamente (Tabela 6).

A condição hídrica apresentou significância estatística aos 240 DAT porém as maiores médias foram observadas para o tratamento com complementação hídrica em todos os períodos avaliados (Tabela 7). Esses resultados mostram que as plantas irrigadas obtiveram maior desenvolvimento foliar.

Albuquerque et al. (2007) verificaram que plantas de pinhãomanso crescidas num espaçamento $4 \mathrm{x} 4$ atingiram área foliar de 2400,6 $\mathrm{cm}^{2}$ aos 150 DAS. Em condições de sequeiro e espaçamento 3 × $2 \mathrm{~m}$, as plantas de pinhão-manso atingiram uma área foliar de $2000 \mathrm{~cm}^{2}$ aos 200 DAS.

Não foram observadas diferenças significativas na produção de frutos das plantas de pinhão-manso para condição hídrica ou espaçamento, tendo havido significância para a interação espaçamento x condição hídrica.

Tabela 4. Número de ramos por planta do pinhão-manso cultivado em diferentes espaçamentos

\begin{tabular}{ccccccccc}
\hline \multirow{2}{*}{ Espaçamento } & \multicolumn{7}{c}{ Número de ramos por planta } \\
\cline { 2 - 9 } & 60 DAT* & 120 DAT & 180 DAT & 240 DAT & 300 DAT & 360 DAT & 420 DAT & 480 DAT \\
\cline { 2 - 9 } & & \multicolumn{7}{c}{ Médias } \\
$2 \times 3$ & $11,79 a$ & $8,93 a$ & $8,83 a$ & $8,37 a$ & $7,95 a$ & $8,00 a$ & $8,00 a$ \\
$2 \times 4$ & $15,25 a$ & $10,54 a$ & $10,12 a$ & $9,45 a$ & $9,08 a$ & $8,91 a$ & $9,00 a$ & $7,91 a$ \\
$3 \times 3$ & $15,12 a$ & $10,83 a$ & $10,08 a$ & $9,41 a$ & $8,91 a$ & $8,62 a$ & $8,62 a$ & $8,62 a$ \\
$3 \times 4$ & $12,45 a$ & $9,83 a$ & $9,62 a$ & $9,12 a$ & $9,12 a$ & $8,91 a$ & $9,04 a$ & $9,04 a$ \\
$4 \times 4$ & $12,58 a$ & $10,04 a$ & $9,37 a$ & $9,00 a$ & $8,62 a$ & $8,54 a$ & $8,50 a$ & $8,50 a$ \\
$5 \times 3$ & $13,87 a$ & $10,20 a$ & $10,08 a$ & $9,33 a$ & $9,00 a$ & $9,04 a$ & $9,04 a$ & $9,04 a$ \\
$5 \times 4$ & $13,20 a$ & $10,83 a$ & $10,58 a$ & $9,79 a$ & $9,41 a$ & $9,33 a$ & $9,33 a$ & $9,33 a$ \\
CV $(\%)$ & 18,56 & 11,06 & 12,02 & 13,82 & 16,78 & 16,90 & 16,52 & 16,71 \\
\hline
\end{tabular}

* DAT - Dias após o transplantio; Médias seguidas de mesma letra minúsculas nas colunas, não diferem entre si pelo teste de Tukey, a nível de 0,05 de probabilidade

Tabela 5. N úmero de ramos por planta do pinhão-manso cultivado em duas condições hídricas

\begin{tabular}{|c|c|c|c|c|c|c|c|c|}
\hline \multirow{3}{*}{ Condição hídrica } & \multicolumn{8}{|c|}{ Número de ramos por planta } \\
\hline & 60 DAT* & 120 DAT & 180 DAT & 240 DAT & 300 DAT & 360 DAT & 420 DAT & 480 DAT \\
\hline & \multicolumn{8}{|c|}{ Médias } \\
\hline $\begin{array}{l}\text { Sequeiro } \\
\text { Comple. Hídrica } \\
\text { CV \% }\end{array}$ & $\begin{array}{l}13,66 \text { a } \\
13,27 \text { a } \\
18,56\end{array}$ & $\begin{array}{l}10,04 \mathrm{a} \\
10,30 \mathrm{a} \\
11,06\end{array}$ & $\begin{array}{l}9,60 \text { a } \\
10,02 \text { a } \\
12,02\end{array}$ & $\begin{array}{r}9,15 \text { a } \\
9,27 \text { a } \\
13,82\end{array}$ & $\begin{array}{c}9,00 \mathrm{a} \\
8,75 \mathrm{a} \\
16,78\end{array}$ & $\begin{array}{c}8,84 \mathrm{a} \\
8,72 \mathrm{a} \\
16,90\end{array}$ & $\begin{array}{c}8,84 \text { a } \\
8,73 \text { a } \\
16,52\end{array}$ & $\begin{array}{r}8,82 a \\
8,73 a \\
16,71\end{array}$ \\
\hline
\end{tabular}

* DAT - Dias após o transplantio; Médias seguidas de mesma letra minúsculas nas colunas não diferem entre si pelo teste de Tukey, a nível de 0,05 de probabilidade

Tabela 6. Área foliar $\left(\mathrm{cm}^{2}\right)$ das plantas do pinhão-manso cultivado em diferentes espaçamentos

\begin{tabular}{|c|c|c|c|c|c|c|c|c|}
\hline \multirow{3}{*}{ Espaçamento } & \multicolumn{8}{|c|}{ Área foliar $\left(\mathrm{cm}^{2}\right)$} \\
\hline & 60 DAT* & 120 DAT & 180 DAT & 240 DAT & 300 DAT & 360 DAT & 420 DAT & 480 DAT \\
\hline & \multicolumn{8}{|c|}{ Médias } \\
\hline $2 \times 3$ & 80,87 a & $94,06 \mathrm{a}$ & $107,97 \mathrm{a}$ & $134,35 \mathrm{a}$ & $73,72 \mathrm{a}$ & $92,83 \mathrm{a}$ & $112,50 \mathrm{~b}$ & $108,16 \mathrm{a}$ \\
\hline $2 \times 4$ & $105,31 \mathrm{a}$ & $103,58 \mathrm{a}$ & $116,67 \mathrm{a}$ & $142,10 \mathrm{a}$ & $97,52 \mathrm{a}$ & $385,98 \mathrm{a}$ & $133,06 a b$ & 94,72 a \\
\hline $3 \times 3$ & 93,11 a & $95,36 \mathrm{a}$ & $107,10 \mathrm{a}$ & $115,91 \mathrm{a}$ & 66,23 a & 96,16 a & $125,82 a b$ & 237,93 a \\
\hline $3 \times 4$ & 91,13 a & $100,09 a$ & 119,73 a & $158,74 \mathrm{a}$ & $76,12 \mathrm{a}$ & $114,03 \mathrm{a}$ & $151,11 \mathrm{a}$ & 99,59 a \\
\hline $4 \times 4$ & 96,38 a & 95,37 a & $128,42 \mathrm{a}$ & $136,21 \mathrm{a}$ & $100,08 \mathrm{a}$ & $116,42 \mathrm{a}$ & $139,45 a b$ & 94,53 a \\
\hline $5 \times 3$ & $103,05 \mathrm{a}$ & 104,04 a & $120,21 \mathrm{a}$ & 155,28 a & 106,03 a & $110,50 a$ & $143,06 a b$ & 94,91 a \\
\hline $5 \times 4$ & 94,69 a & 96,82 a & 117,13 a & 138,38 a & 84,12 a & 124,85 a & 154,63 a & $104,56 \mathrm{a}$ \\
\hline CV \% & 23,40 & 18,45 & 18,84 & 20,93 & 37,77 & 172,63 & 14,57 & 108,19 \\
\hline
\end{tabular}

* DAT - Dias após o transplantio; Médias seguidas de mesma letra minúsculas nas colunas não diferem entre si pelo teste de Tukey, a nível de 0,05 de probabilidade 
Tabela 7. Área foliar $\left(\mathrm{cm}^{2}\right)$ das plantas do pinhão-manso cultivado em duas condições hídricas

\begin{tabular}{ccccccccc}
\hline & \multicolumn{7}{c}{ Área foliar $\left(\mathbf{c m}^{2}\right)$} \\
\cline { 2 - 8 } Condição hídrica & 60 DAT* & 120 DAT & 180 DAT & 240 DAT & 300 DAT & 360 DAT & 420 DAT & 480 DAT \\
\cline { 2 - 9 } & \multicolumn{8}{c}{ Médias } \\
Sequeiro & $94,73 \mathrm{a}$ & $95,97 \mathrm{a}$ & $112,60 \mathrm{a}$ & $126,33 \mathrm{~b}$ & $78,12 \mathrm{a}$ & $116,14 \mathrm{a}$ & $131,11 \mathrm{a}$ & $99,03 \mathrm{a}$ \\
Comple. Hídrica & $95,13 \mathrm{a}$ & $100,98 \mathrm{a}$ & $120,86 \mathrm{a}$ & $153,95 \mathrm{a}$ & $94,40 \mathrm{a}$ & $181,23 \mathrm{a}$ & $143,07 \mathrm{a}$ & $139,38 \mathrm{a}$ \\
CV \% & 23,40 & 18,45 & 18,84 & 20,93 & 37,77 & 172,63 & 14,57 & 108,19 \\
\hline
\end{tabular}

* DAT - Dias após o transplantio; Médias seguidas de mesma letra minúsculas nas colunas não diferem entre si pelo teste de Tukey, a nível de 0,05 de probabilidade

Observando o desdobramento da interação espaçamento $\mathrm{x}$ condição hídrica (Tabela 8), verifica-se que em condições de sequeiro não houve diferença entre os espaçamentos, o mesmo se verificando para as condições de irrigação. Por outro lado se nota, comparando-se condições hídricas, diferença estatística apenas nos espaçamentos 3 x 3 e 4 x 4, com uma produção 2,43 vezes maior para a condição de sequeiro quando se usa um espaçamento de $3 \times 3$; já para o espaçamento de $4 \times$ 4 a condição de irrigação produz 3,10 vezes mais que a de sequeiro (Tabela 8 ).

Tabela 8. D esdobramento da interação para a produção de sementes das plantas de pinhão-manso cultivado em diferentes espaçamentos entre sulcos e irrigação

\begin{tabular}{cccc}
\hline \multirow{2}{*}{ Espaçamento } & \multicolumn{3}{c}{ Produção de sementes (g) } \\
\cline { 2 - 4 } $2 \times 3$ & Sequeiro & Irrigado & Medias \\
$2 \times 4$ & $759,13 \mathrm{aA}$ & $393,41 \mathrm{aA}$ & 326,27 \\
$3 \times 3$ & $832,17 \mathrm{aA}$ & $384,30 \mathrm{aA}$ & 562,94 \\
$3 \times 4$ & $373,32 \mathrm{aA}$ & $342,77 \mathrm{aB}$ & 587,47 \\
$4 \times 4$ & $243,18 \mathrm{aB}$ & $753,84 \mathrm{aA}$ & 475,08 \\
$5 \times 3$ & $479,94 \mathrm{aA}$ & $449,33 \mathrm{aA}$ & 498,42 \\
$5 \times 4$ & $503,51 \mathrm{aA}$ & $296,91 \mathrm{aA}$ & 464,63 \\
\hline Medias & 490,40 & 456,75 \\
\hline CV $\%$ & \multicolumn{4}{c}{46,83} \\
\hline
\end{tabular}

* DAT - Dias após o transplantio; Médias seguidas de mesma letra minúsculas entre linhas maiús culas entre colunas não diferem entrei si pelo teste de Tukey, a nível de 0,05 de probabilidade

No semiárido paraibano não foi observada influência do sistema de irrigação sobre a produção dos frutos de pinhãomanso ainda no primeiro ano de cultivo (Dantas Neto et al., 2009).

A produtividade pode variar de acordo com a região, o método de cultivo, o manejo dos tratos culturais e a intensidade das chuvas, tendo o potencial de produtividade do pinhão-manso em sementes se tornado evidente pelas produções entre 6.373 e $6.468 \mathrm{~kg} \mathrm{ha}^{-1}$ das melhores plantas, no primeiro ciclo de colheita (Arruda et al., 2004). Tais valores diferem dos encontrados neste trabalho em que a maior produtividade foi de $1808,44 \mathrm{~kg} \mathrm{ha}^{-1}$ no espaçamento de $2 \times 4$, em sequeiro (Figura 1).

Lima et al. (2009) afirmam que se pode alcançar produtividade de $500 \mathrm{~kg} \mathrm{ha}^{-1}$ de semente na primeira colheita e citam, ainda, que na segunda colheita se pode obter produtividade de 975 $\mathrm{kg} \mathrm{ha}^{-1}$, permitindo afirmar que o pinhão-manso pode vir a ser uma fonte de matéria-prima para produção de biodiesel. Segundo Nunes et al. (2008), no primeiro ano de cultivo, no oeste da Bahia, a produtividade ficou em torno de $713,82 \mathrm{~kg} \mathrm{ha}^{-1}$, próximo do mínimo recomendado para a mamona visando a uma lucratividade satisfatória.

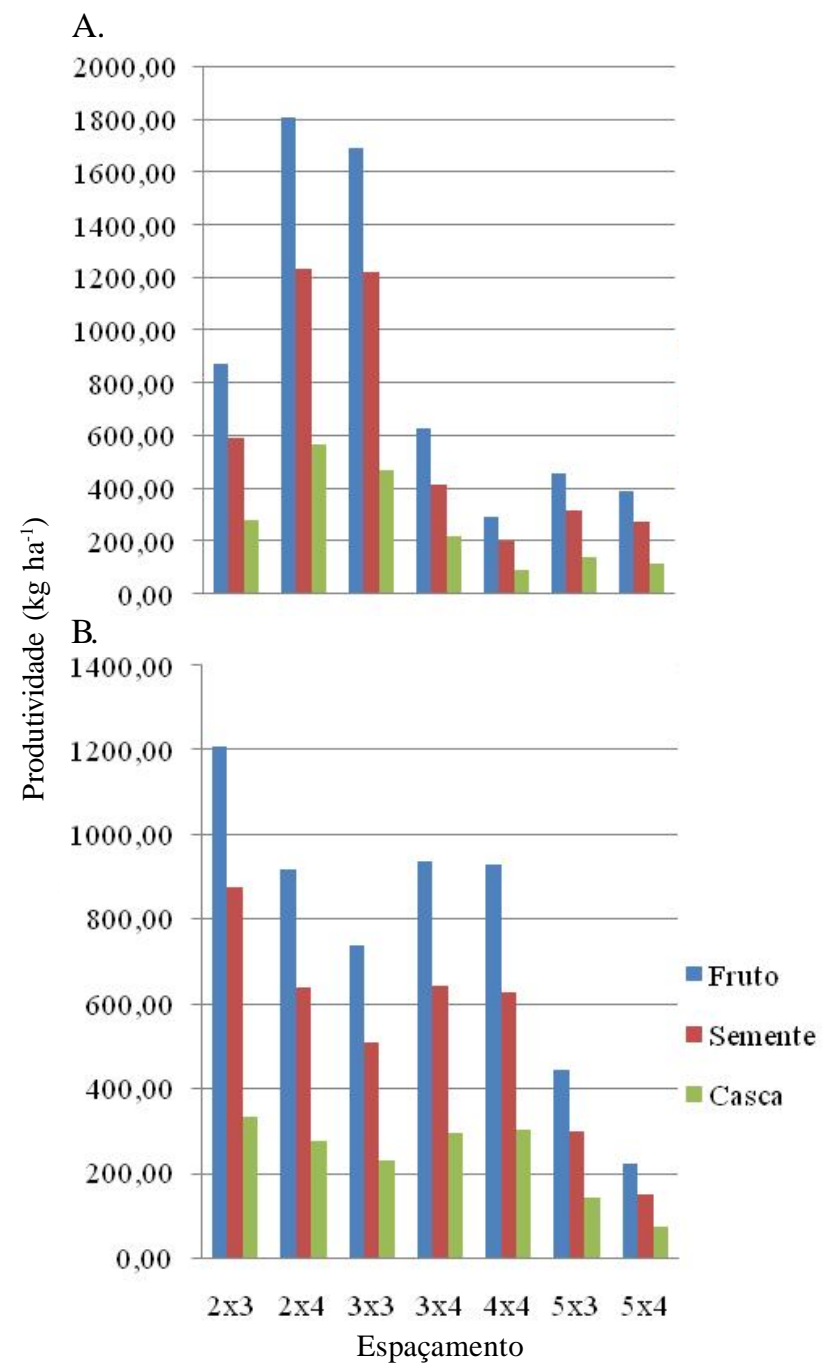

Figura 1. Produtividade do pinhão-manso em sequeiro (A) e irrigado (B)

Segundo Drumond et al. (2010), nos nove primeiros meses de implantação da cultura do pinhão-manso na região de Petrolina, PE, com espaçamento de $2 \times 2 \mathrm{~m}$, a produtividade média de sementes e frutos das plantas irrigadas foi de 871 $\mathrm{kg} \mathrm{ha}^{-1}, 3,5$ vezes maior que aquela obtida apenas com o regime normal de chuva, de $246 \mathrm{~kg} \mathrm{ha}^{-1}$.

Duarte (2009) verificou, estudando o efeito da densidade e época do ano sobre a produtividade de pinhão-manso, que

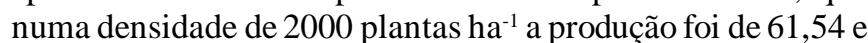
$251,42 \mathrm{~kg} \mathrm{ha}^{-1}$ de frutos aos 395 e 449 DAS, respectivamente e, para uma população de 1500 plantas, a produtividade média foi de 50,14 $\mathrm{kg} \mathrm{ha}^{-1}$ aos 416 DAS.

Como a produção de sementes por área está diretamente relacionada à densidade, para se obter uma produtividade maior 
por área no tratamento em sequeiro, o espaçamento de 2 x $4 \mathrm{~m}$ com 2.222 plantas ha-1 é o recomendado, com produção inicial de $1808,44 \mathrm{~kg} \mathrm{ha}^{-1}$ de fruto: $1236,61 \mathrm{~kg} \mathrm{ha}^{-1}$ de sementes e 581,83 $\mathrm{kg} \mathrm{ha}^{-1}$ de casca (Figura 1A).

No tratamento irrigado com espaçamento $2 \times 3 \mathrm{~m}, 2.963$ plantas ha-1 ${ }^{-1}$ apresentaram maior produção de frutos: 1208,41 $\mathrm{kg} \mathrm{ha}^{-1}$ de sementes e $874,27 \mathrm{~kg} \mathrm{ha}^{-1}$ de casca.

A intensidade de aplicação de água $\left(\mathrm{Ia}, \mathrm{mm} \mathrm{h}^{-1}\right)$ e o coeficiente de uniformidade de Christiasen (CUC, \%) foram reduzidos ao longo do experimento sendo observados $\mathrm{Ia}=1,0$ $\mathrm{mm} \mathrm{h}^{-1}$ e CUC $=91,3 \%$ no início do experimento e Ia $=0,65$ $\mathrm{mm} \mathrm{h}^{-1}$ e CUC $=79,65 \%$ no final do experimento. Esta redução ocorreu, provavelmente, pela qualidade da água de irrigação, rica em algas e material particulado que não foi adequadamente retido no sistema de filtragem (filtro de disco de 120 mesh).

O período mais crítico para o manejo da irrigação se situou entre agosto e setembro de 2010 em que foram observados os maiores valores de ETo.

\section{ConclusõEs}

1. O espaçamento não influenciou na altura de plantas, ramificação, área foliar nem na produtividade do pinhão-manso.

2. A complementação hídrica influenciou a altura de plantas a partir dos 240 dias após o transplantio das mudas de pinhãomanso.

3. A complementação hídrica não foi significativa para ramificação e produtividade.

4. A produtividade apresentou diferença significativa em sequeiro no espaçamento 3 x 3 e $3 \times 4$ com complementação hídrica.

\section{LITERATURA CITADA}

Albuquerque, W. G.; Severino, L. S.; Beltrão, N. E. de M.; Freire, M. A. de O.; Nascimento, J. J. V. R. do; Crescimento do pinhão nitrogenada manso ( Jatropha curcas L.) em função de níveis de água e adubação. In: Congresso Brasileiro de Mamona: Energia e Ricinoquímica.3, 2007, Salvador. Resumos... Salvador: Embrapa - Algodão, 2007. 118p.

Arruda, F. P. de; Beltrão, N. E. de M.; Andrade, A. P. de; Pereira, W. E.; Severino, L. S. Cultivo do pinhão-manso (Jatrofa curcas L.) como alternativa para o semiárido Nordestino. Revista Brasileira de Oleaginosas e Fibrosas, v.8, p.789-799, 2004.

Bernardi, G.; Findley, L.; Olivares, A. R. Vicariance and dispersal across Baja California in disjunct marine fish populations. Evolution, v.57, p.1599-1609, 2003.

Chaves, L. H. G.; Cunha, T. H. C. S.; Barros Júnior, G.; Lacerda, R. D. de; Dantas Júnior, E. E. Zinco e cobre em pinhão manso. 1. Crescimento inicial da cultura. Revista Caatinga, v.22, p.9499, 2009.

Dantas Neto, J. D,; Trajano, E. V. de A.; Araújo, B. A. de; Santos, Y. M. dos; Azevedo, C. A. V. de. Crescimento e produção do pinhão-manso irrigado sob diferentes adubações no semiárido paraibano. In: Congresso Brasileiro de Pesquisas de Pinhão-Manso, 1, 2009, Brasília. Anais... Brasília: EMBRAPA, 2009. CD Rom
Drumond, M. A.; Santos, C. A. F.; Oliveira, V. R.; Martins, J. C.; Anjos, J. B.; Evangelista, M. R. V. Desempenho agronômico de genótipos de pinhão manso no semiárido pernambucano. Ciência Rural, v.40, p.44-47, 2010.

Duarte, A. O peso da matéria-prima. Biodieselbr, n.9, 19p, 2009.

Evangelista, A. W. P.; Melo, P. C.; Oliveira, E. L.; Faria, M. A. Produtividade e rendimento de sementes de pinhão-manso. Engenharia Agrícola, v.31, p.315-323, 2011.

Francis, G.; Edinger, R.; Becker, K. A concept for simultaneous wasteland reclamation, fuel production, and socio-economic development in degraded areas in India: Need, potential and perspectives of Jatropha plantations. Natural Resources Forum, v.29, p.12-24, 2005.

Genuchten, M. T. van. A closed form equation for predicting the hydraulic conductivity of unsaturated soils. Soil Science Society of America Journal, v.44, p.892-898, 1980.

Genuchten, M. T. van. A closed form equation for predicting the hydraulic conductivity of unsaturated soils. Soil Science Society of America Journal, v.44, p.892-898, 1991.

Horschutz, A C O; Magalhaes, F M.; Branquinho, R. G.; Lopes, A. B. O.; Alves, J. M.; Efeitos do volume de substrato, saturação por bases e matéria orgânica na produção de mudas de pinhão-manso. In: Congresso de Iniciação Científica, 2 Semana do Meio Ambiente, 2008. Rio Verde: CEFET, Anais... 2008 CD Rom.

Laviola, B. G.; Dias, L. A. dos S. Teor e acúmulo de nutrientes em folhas e frutos de pinhão-manso. Revista Brasileira de Ciência do Solo, v.32, p.1969-1975, 2008.

Lima, R. L. S.; Severino, L. S.; Sampaio, L. R.; Freire, M. A. O.; Beltrão, N.E.M.; Arriel, N. H. C. Crescimento e teor foliar de nutrientes em mudas de pinhão-manso (Jatropha curcas L.) em substratos contendo cinco materiais orgânicos e fertilizante mineral. Revista de Oleaginosas e Fibrosas, v.13, p.29-36, 2009.

Nery, A. R.; Rodrigues, L. N.; Silva, M. B. R. da; Fernandes, P. D.; Chaves, L. H. G.; Dantas Neto, J.; Gheyi, H. R. Crescimento do pinhão-manso irrigado com águas salinas em ambiente protegido. Revista Brasileira de Engenharia Agrícola eAmbiental, v.13, p.551-558, 2009.

Nunes, C. F.; Pasqual, M.; Santos, D. N. dos; Custódio, T. N.; Araújo, A. G. de. Diferentes suplementos no cultivo in vitro de embriões de pinhão manso. Pesquisa Agropecuária Brasileira, v.43, p.9-14, 2008.

Martins, C. C.; Machado, C. G.; Cavasini, R. Desenvolvimento inicial de mamona e pinhão-manso em solo submetido a diferentes corretivos e doses de fósforo. Revista Verde de Agroecologia e Desenvolvimento Sustentável, v.5, p.143$150,2010$.

Oliveria, R. B. de; Gimenez, V. M. M.; Godoy, S. A. P. de. Intoxicações com espécies da família Euphorbiaceae. Revista Brasileira de Biociências, v.50, p.69-71, 2007.

Openshaw, K. A. Review of Jatropha curcas: An oil plant of unfulfilled promise. Biomass and Bioenergy, v.19, p.1-15, 2000.

Pinto Júnior, A; Almeida, P. F; Desenvolvimento inicial do pinhão-manso em diferentes espaçamentos. In: Congresso Brasileiro de Pesquisas de Pinhão Manso, 1, 2009, Brasília. Anais... Brasília: EMBRAPA, 2009. CD Rom 
Ratree, S. A preliminary study on physic nut (Jatropha curcas L.) in Thailand. Pakistan Journal of Biogical Sciences, v.7, p.1620-1623, 2004.

Santos, Z. M. dos. Caracteres agronômicos da cultura do pinhão-manso (Jatropha curcas L.) em diferentes espaçamentos, adubações e poda. Campos dos Goytacazes: UFRRJ, 66p. 2010. Dissertação Mestrado

Schiavo, J. A.; Silva, C. A.; Rosset, J. S.; Secretti, M. L.; Sousa, R. A. C.; Cappi, N. Composto orgânico e inoculação micorrízica na produção de mudas de pinhão-manso. Pesquisa Agropecuária Tropical, v.40, p.322-329, 2010.
Severino, L. S.; Vale, L. S.; Beltrão, N. E. de M. A simple method for measurement of Jatropha curcas leaf area. Revista Brasileira de Oleaginosas e Fibrosas, v.11, p.914, 2007.

Sujatha, M.; Makkar, H. P. S.; Becker, K. Shoot bud proliferation from axillary nodes and leaf sections of nontoxic Jatropha curcas L. Plant Growth Regulation, v.47, p.83-90, 2009.

Teixeira L. C. Potencialidades de oleaginosas para produção de biodiesel. Revista Informe Agropecuário, v.26, p.18-27, 2005. 